\title{
Thin-film polyetherimides with controlled refractive indices
}

\author{
Ramil Mercado, Yubao Wang, Tony Flaim, William DiMenna, and Udayan Senapati \\ Brewer Science, Inc., 2401 Brewer Dr., Rolla, MO 65401 USA
}

\begin{abstract}
A series of soluble, fully aromatic polyetherimides were prepared as candidate materials for optical coating applications. Most of the new polymer coatings possessed high transparency in the optical and near-infrared spectral regions at thicknesses ranging from 1 to 10 microns. The refractive indices obtained ranged from 1.60 to 1.80 at visible wavelengths, with the highest values generally being obtained near $400 \mathrm{~nm}$ followed by a gentle decline as wavelength increased to $700 \mathrm{~nm}$ and beyond. The refractive index values could be controlled by varying the dianhydride and diamine composition. All of the polyimides showed good thermal stability to $400^{\circ} \mathrm{C}$ and displayed glass transition temperatures above $220^{\circ} \mathrm{C}$, making them excellent candidates for device applications where increased refractive index and high optical clarity are desired. The paper will discuss the preparation and physical and optical properties of the polymers and compare them to other high index coating systems.
\end{abstract}

\section{INTRODUCTION}

Polymers having high indices of refraction have immense applications in optics and photonics due to their ability to reduce reflection losses at interfaces and, hence, increase light output. The use of semiconductor materials in photonics sources, detectors, and devices has increased the requirements for high index polymers in applications ranging from light-emitting diodes (LEDs) to planar light wave circuits. Most semiconductor materials have refractive indices in the range of 2.5 to 3.5 . Optically clear polymers or epoxies that could significantly reduce reflection losses at the semiconductor-air interface do not exist because the index of refraction of most optical polymers or epoxies are in the range of 1.5 to 1.55 . High-brightness LEDs have garnered much interest lately for myriad applications and potentially as replacement white light sources. Gallium nitride blue LEDs are typically grown on sapphire or silicon carbide substrates and as a result need some high refractive index medium between the chip and the lens to increase the light extraction efficiency. A polymeric material with an index of refraction in the range of 1.7 to 1.8 could definitely increase the light output from such packaged LED sources. Further, for most integrated optics or planar photonic circuits, the material of choice on semiconductor materials such as silicon, indium phosphide, and gallium arsenide is a high index polymer with high index contrast because of optimized losses and the capability of tuning. In addition, the mechanical characteristics of polymers allow them to be processed by unconventional techniques such as molding, casting, stamping, embossing, etc., which lead to rapid low-cost shaping for waveguide formation. High index polymers can also be used for beam splitting applications in diffraction gratings. The high index of refraction would decrease the optical thickness of the gratings, thus facilitating thinner grating structures. Because these materials can be spun in layer thicknesses ranging from a few hundred nanometers to as high as 10 microns, these high index polymers can be used as antiglare coatings for eyeglasses and other ophthalmic applications.

We have previously reported a new class of amorphous aromatic polyetherimides that possess high refractive indices (1.62 to 1.78) at visible wavelengths. These solvent-soluble polyetherimides are eminently suitable for optical applications due to their excellent optical clarity at wavelengths greater than $400 \mathrm{~nm}$. Also, the polymers are already imidized during the synthesis process, which provides additional advantages for optical device applications in that the high refractive indices are already "built-in" and the coatings only require complete solvent removal and in that the formation of voids that contribute to light scattering is avoided. Sensitivity to moisture and variations in temperature, as is typical in polyimide precursor solutions, are also precluded. These polymers also possess the usual characteristics of aromatic polyimides, such has high glass transition temperatures $\left(>225^{\circ} \mathrm{C}\right)$, thermal stability $(<5 \%$ weight loss at $400^{\circ} \mathrm{C}$ ) and good mechanical strength. Thicknesses ranging from 1 to 10 microns were obtained with these polyetherimide systems. They were also easily coated on to substrates such as glass, quartz, and silicon with good adhesion.

Copyright 2004 Society of Photo-Optical Instrumentation Engineers. This paper will be published in Proceedings of SPIE: Organic Photonic Materials and Devices VI, vol. 5351, James G. Grote and Toshikuni Kaino, eds., 2004, and is made available as an electronic preprint with permission of SPIE. One print or electronic copy may be made for personal use only. Systematic or multiple reproduction, distribution to multiple locations via electronic or other means, duplication of any material in this paper for a fee or for commercial purposes, or modification of the content of the paper are prohibited. 
In expanding the family of these solvent-soluble, pre-imidized polymer systems, we have found that the refractive indices can be effectively tailored by the judicious choice of dianhydride and/or diamine components of the polymers.

\section{EXPERIMENTAL}

The polyetherimides are preferentially synthesized by separately dissolving the dianhydride and the diamine in $\mathrm{N}, \mathrm{N}$-dimethyacetamide (DMAc) or N-methylpyrrollidinone (NMP). The poly(amic acid) is then generated by adding the dianhydride solution to the diamine solution and mixing for 24 hours. Thermal imidization is then done by refluxing the poly(amic acid) solution for about 24 hours with the use of an azeotroping solvent such as toluene to distill off the water that is generated. This synthetic scheme, as applied to OptiNDEX ${ }^{\mathrm{TM}}$ B44, is shown in Figure 1.
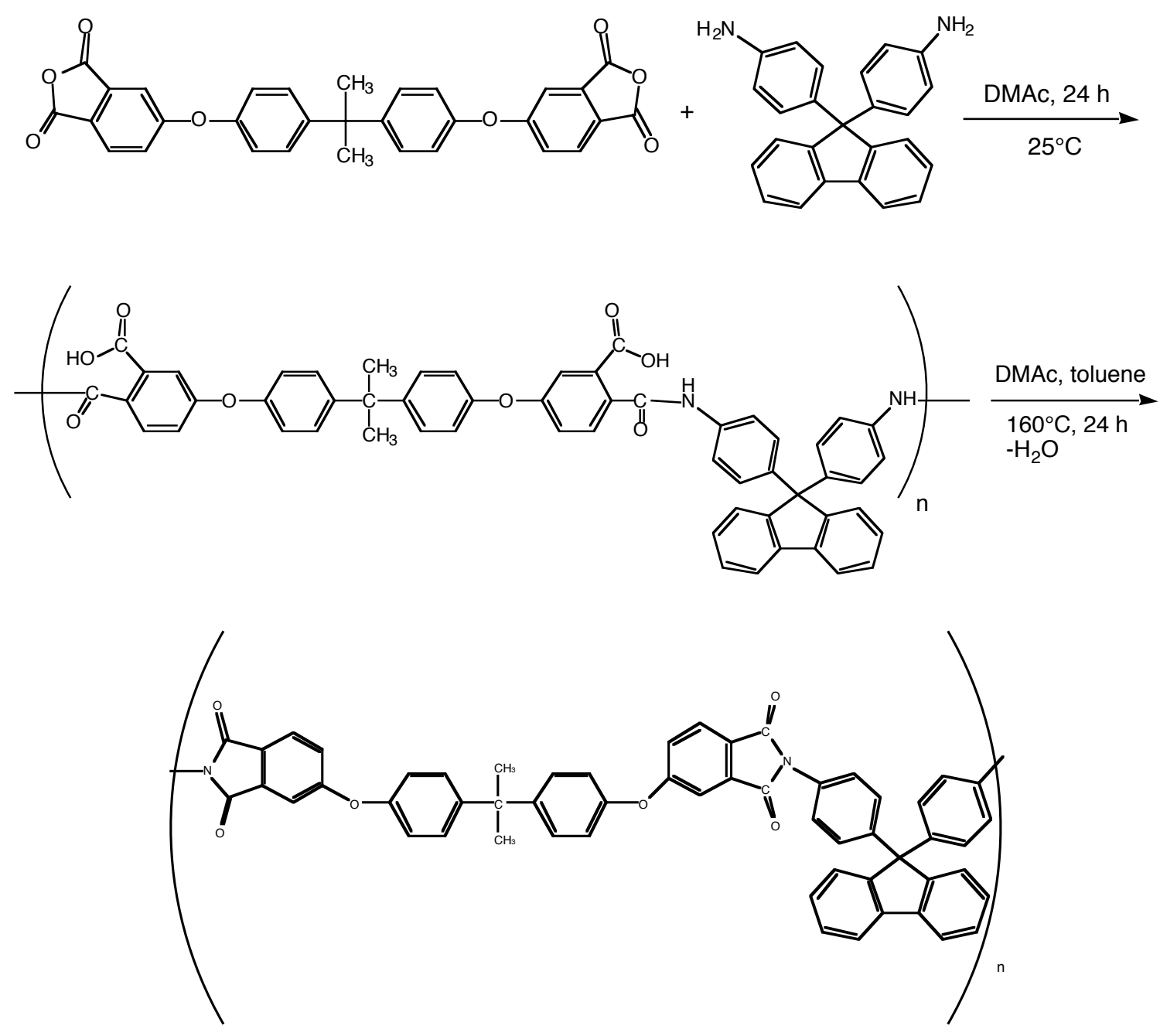

Figure 1. Scheme for OptiNDEX ${ }^{\mathrm{TM}}$ B44 synthesis.

An alternative synthesis may also be done using the ester-acid method disclosed by Eichstadt and coworkers [1]. In this method, the dianhydride is made to react with an excess of an alcohol, such as ethyl alcohol, to form the ester-acid. The excess alcohol is distilled off, the diamine solution in NMP is added, and the resulting solution is heated to $190^{\circ} \mathrm{C}$ in the presence of $o$-dichlorobenzene as an azeotroping solvent. 
The polyimide that is formed is then purified by precipitation into a suitable non-solvent such as methanol, and then dried in a vacuum oven to remove residual solvents. The polyimides obtained have molecular weights (from gel permeation chromatography using a Brewer Science, Inc., proprietary solvent mixture and measured against polystyrene standards) ranging from 50,000 to 100,000 daltons. The polyimide solutions are then prepared by dissolving the recovered solids in a cyclic aliphatic ketone, such as cyclopentanone or cyclohexanone in concentrations ranging from $15 \%$ to $25 \%$ by weight. The resulting yellow- or amber-colored solutions are filtered through a 0.2 -micron filter to remove any particles prior to coating.

The coating solutions were applied onto glass, quartz, or silicon substrates by spin coating at 500 to $5000 \mathrm{rpm}$ for 80 seconds. A soft bake is performed on a $100^{\circ} \mathrm{C}$ hotplate for 2 minutes followed by a final bake on a $205^{\circ} \mathrm{C}$ hotplate for 1 minute to ensure the complete removal of residual solvent. The thickness of each coating was then measured with a Geartner elipsometer or an Alpha-Step profilometer. Coating transparency, reported as percent transmission (\% T) for a given film thickness, was measured with a Cary recording UV-visible spectrophotometer, with no corrections being made for scattering or reflective losses. The refractive index of each coating was determined with the aid of a J.A. Woolam variable angle spectroscopic ellipsometer (VASE).

\section{RESULTS}

\subsection{Refractive indices}

In 2003, the authors reported the first representatives of this class of solvent-soluble aromatic polyetherimides [2]. The first example was OptiNDEX ${ }^{\mathrm{TM}}$ B38 (BPADA/BAPS, Fig. 2) with a reported index ranging from 1.68 to 1.74 in the region from $700 \mathrm{~nm}$ to $400 \mathrm{~nm}$. The other example, OptiNDEX ${ }^{\mathrm{TM}}$ B44 (BPADA/FDA, Fig. 3) had a reported index range of 1.69 to 1.79 from $700 \mathrm{~nm}$ to $400 \mathrm{~nm}$. Using these results as a guide, several other polyetherimide systems were synthesized with the aim of providing specific refractive index ranges in the visible spectrum for a certain polyetherimide system. These polymers all had to be solvent-soluble and processable in the imidized form, as well as to provide a reasonable amount of transparency $(\geq 80 \%)$ in the visible region.

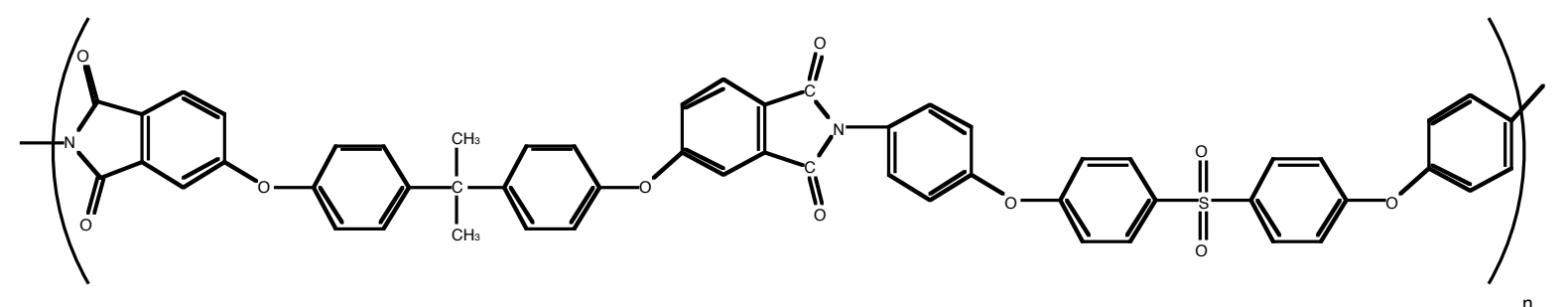

Figure 2. Representative structure of OptiNDEX ${ }^{\mathrm{TM}}$ B38.

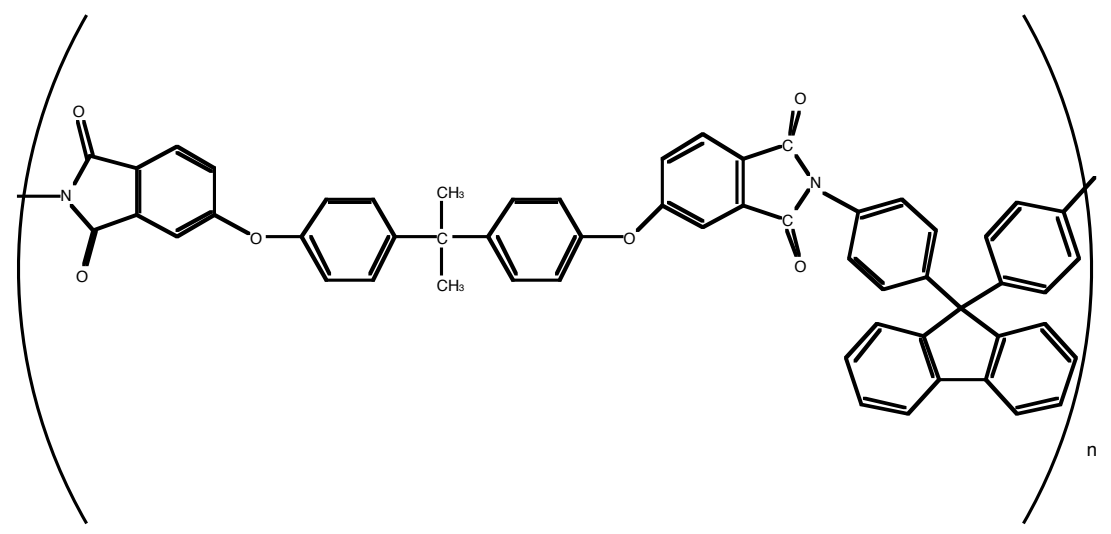

Figure 3. Representative structure of OptiNDEX ${ }^{\mathrm{TM}}$ B44. 
The following chart (Fig. 4) shows the comparative refractive index values for four other polyetherimide systems, in addition to the two OptiNDEX samples reported previously, at various wavelengths. Not surprisingly, the two fluorinated examples both show lower refractive indices across the spectrum compared to the polyetherimides without fluorine atoms. However, an unexpected result is that the polyetherimide BPADA/mBAPS shows virtually the same refractive index values as OptiNDEX B38 (BPADA/BAPS). As a general rule, higher refractive indices are obtained by utilizing the fluorenyl-containing systems. However, the obtained refractive indices for OptiNDEX ${ }^{\mathrm{TM}} \mathrm{B} 44$ are higher than what can be expected simply from the use of aromatic fluorenyl units because its refractive index curve is even higher than BPFPA/FDA, which has double the amount of fluorenyl groups.

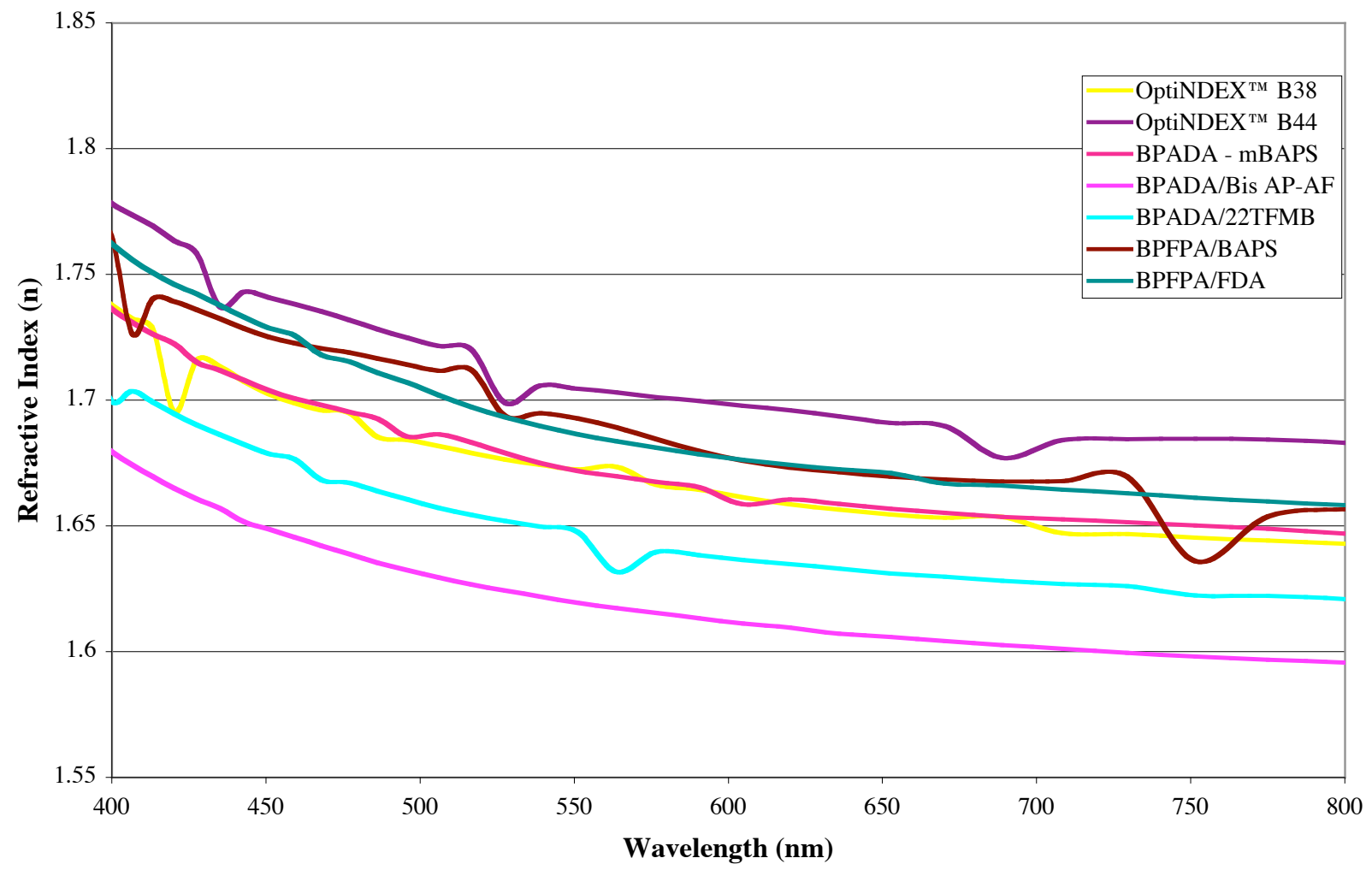

Figure 4. Comparative chart of polyetherimide refractive indices. 


\section{Transmission Curves of Selected Polyimides}

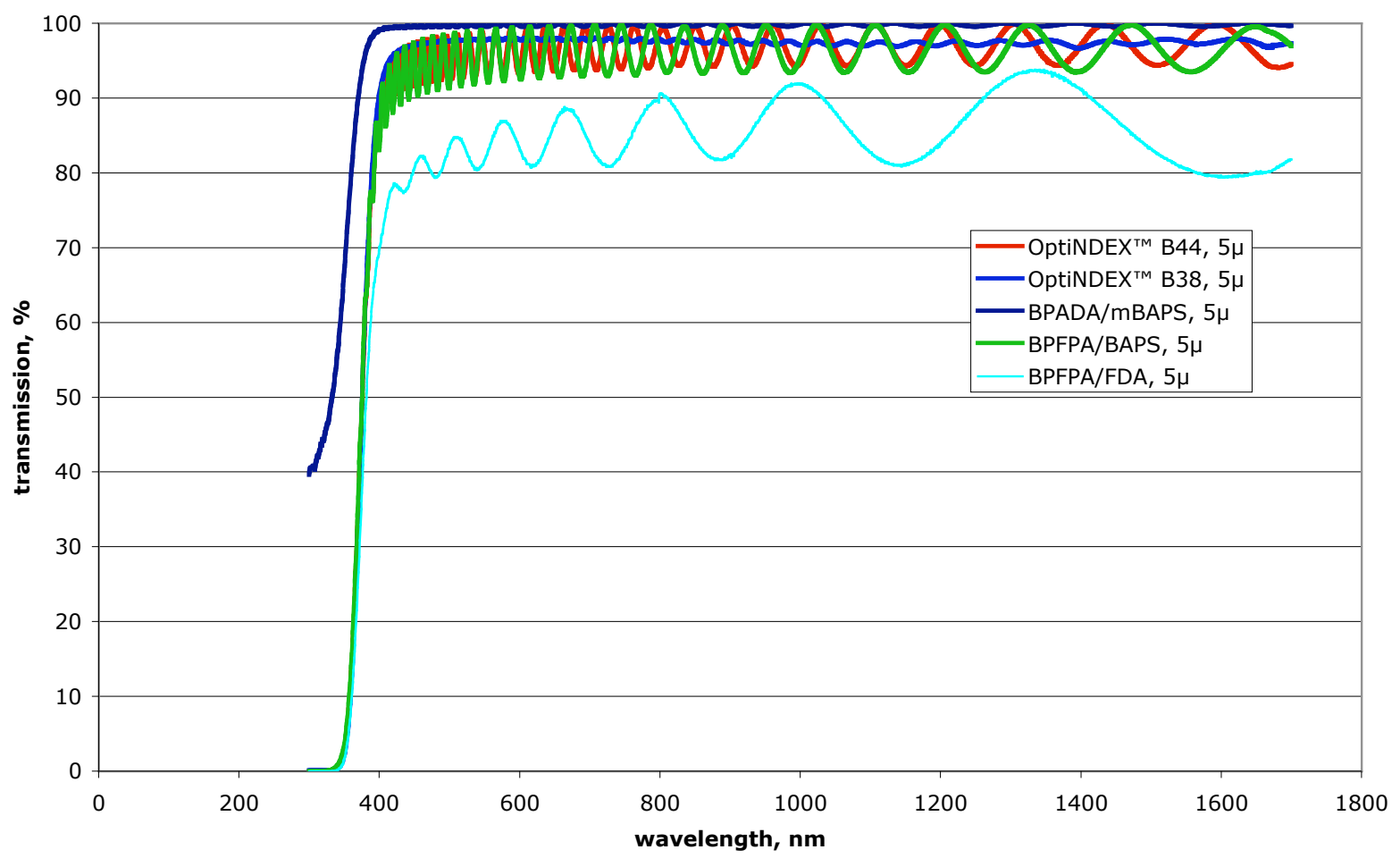

Figure 5. Comparative UV-visible transmission curve for selected polyetherimides.

Figure 5 shows the transmission curves of several of the selected polyetherimide systems. As seen before, both OptiNDEX ${ }^{\mathrm{TM}}$ B38 and B44 show excellent transmission in the visible range, exceeding $80 \%$ at $400 \mathrm{~nm}$ and more than $90 \%$ from $420 \mathrm{~nm}$ onwards into the near-infrared region. BPFPA/BAPS, as seen from the chart, mirrors this behavior closely. However, a marked decrease in transmission is shown by the BPFPA/FDA polymer, at the expense of its increased refractive index over this range. Surprisingly, the BPADA/mBAPS polymer shows a vast improvement in transparency over OptiNDEX ${ }^{\mathrm{TM}}$ B38, achieving more than $99 \%$ transmission in the visible to near-IR range, and more than $90 \%$ transmission in the near ultraviolet (to $370 \mathrm{~nm}$ ). Transmission curves for the two fluorinated examples BPADA/22TFMB and BPADA/BisAPAF were not reported due to the inability to obtain good coatings of sufficient thicknesses. However, it is widely known that fluorinated polyimides have highly polarized carbon-fluorine bonds pulling electron density away from the rest of the polymer chain and therefore have excellent optical and near-UV transmission due to lower charge transfer interactions.

\section{DISCUSSION}

Many aromatic polyimides often suffer from poor solubility and are generally intractable [3]. We have attempted to address these issues by incorporating highly flexible linker groups such as ether, sulfone, or isopropylidene groups (or a combination thereof) in the backbone. The solubility can also be improved by the use of a bulky organic group that is pendant to the main chain, in this case trifluoromethyl (BPADA/BisAPAF and BPADA/22TFMB) and fluorenyl groups $\left(\right.$ OptiNDEX ${ }^{\mathrm{TM}}$ B44, BPFPA/BAPS, and BPADA/FDA). These allow the synthesis and application of the polyetherimides from solution themselves instead of solutions of the poly(amic acid) precursors. Thus the formation of voids from the evolution of water from bulk imidization is avoided, which leads to excellent optical film properties [4]. 
Also, there is no longer the necessity of having to process the polymer films at high temperatures $\left(>230^{\circ} \mathrm{C}\right)$, the requisite processing only being what is necessary to eliminate solvent.

\subsection{Structure-property relationships}

Generally, many polyimides, especially fully aromatic polyimides, have relatively high refractive indices compared to other organic polymers. This is true even for fluorinated derivatives, as shown by the examples presented in this work. Both BPADA/22TFMB and BPADA/BisAPAF have indices greater than 1.65 at $400 \mathrm{~nm}$. It is believed that between the two polymer systems, the higher indices reported for BPADA/BisAPAF result from the position of the trifluoromethyl groups. In BPADA/BisAPAF, the $-\mathrm{CF}_{3}$ units are pendant on the bridging isopropylidene group, leading to a lower net electron-withdrawing effect on the aromatic groups. The phenyl rings retain much of their electron density (and polarizability) compared to $\mathrm{BPADA} / 22 \mathrm{TFMB}$, where the $-\mathrm{CF}_{3}$ units are directly on the phenyl rings and thus pull electron density away from the ring. And yet the refractive indexes are high for both polymers because of the number of aromatic and imide groups in the repeat units.

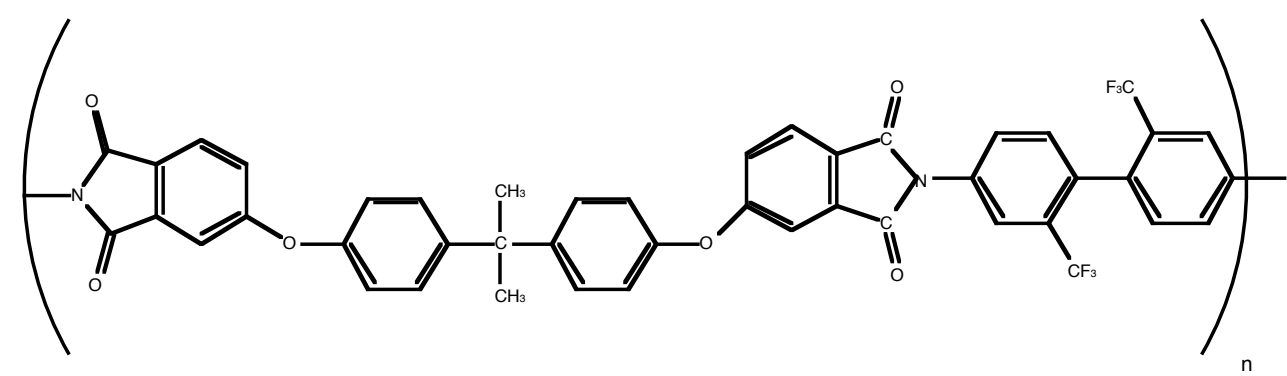

BPADA/22TFMB

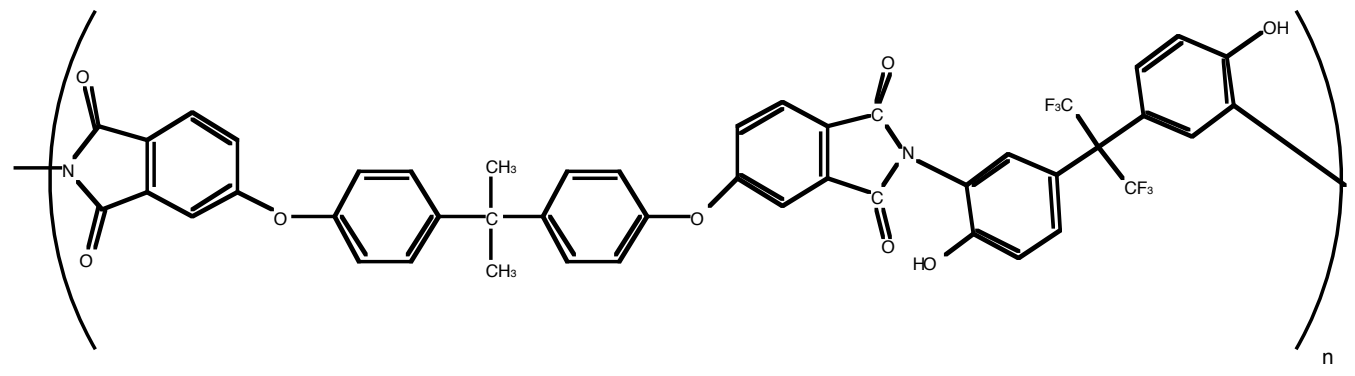

BPADA/BisAPAF

The absence of electron-withdrawing trifluoromethyl groups leads to higher refractive indices in aromatic polyetherimides. In addition, the higher density or concentration of aromatic groups should also lead to higher indices. This general trend is followed in the following examples. 
<smiles>CN1C(=O)c2ccc(Oc3ccc(C(C)(C)c4ccc(Oc5ccc(C(C)(C)c6ccc(Oc7ccc(Oc8ccc(O)cc8)cc7)cc6)cc5)cc4)cc3)cc2C1=O</smiles>

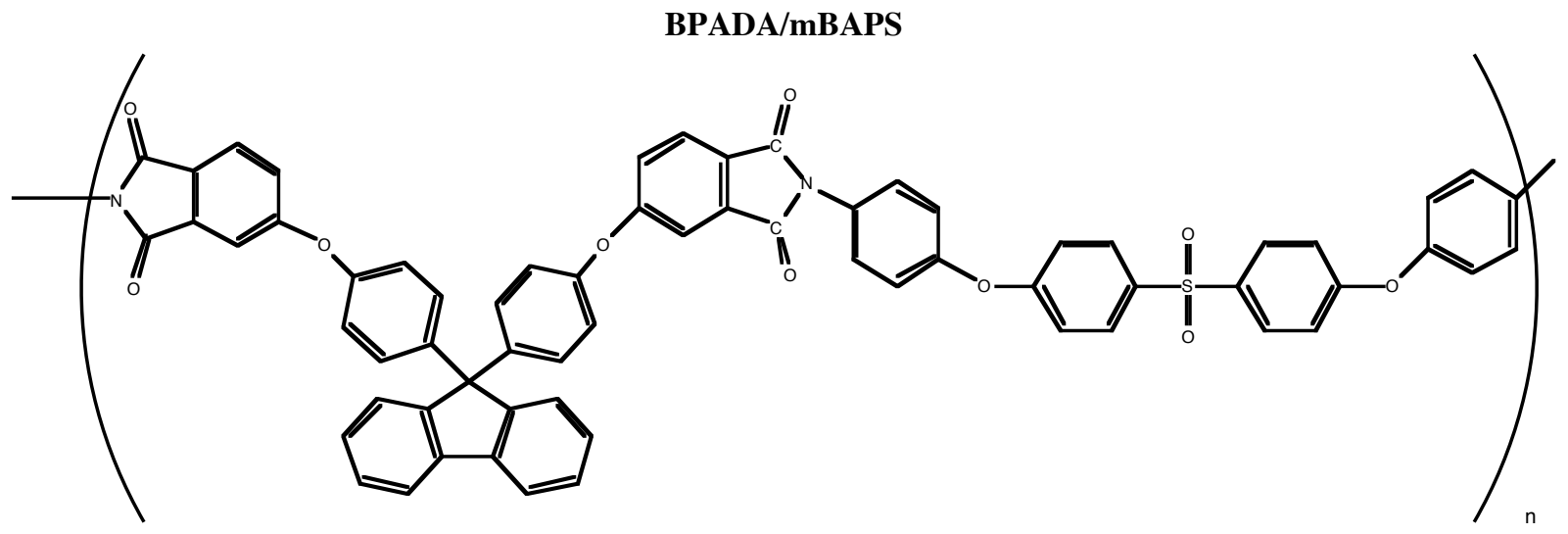

BPFPA/BAPS

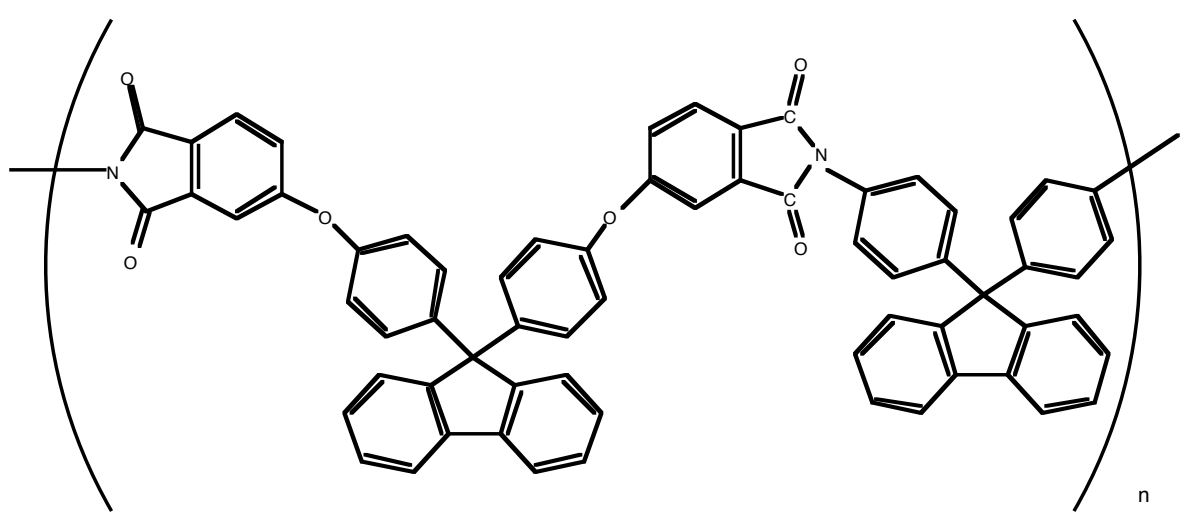

BPFPA/FDA

The refractive index of BPADA/mBAPS is comparable to its para-analogue, OptiNDEX ${ }^{\mathrm{TM}}$ B38. Presumably, in these two systems, index is largely determined by the polarizability of the functional groups from within the chain and not by the formation of interchain and/or intrachain charge-transfer complexes. Otherwise, the expected conformational differences would reflect significantly different refractive indices for the two isomers.

The fluorenyl-containing polyetherimides all reflect relatively higher index values, presumably from the comparatively high concentration of $\pi$-electrons in their repeating units. It is notable, however, that BPFPA/FDA does not show higher indices compared to OptiNDEX ${ }^{\mathrm{TM}} \mathrm{B} 44$, despite the two fluorenyl groups in its repeat unit. This apparent anomalous behavior cannot be explained simply by molecular composition alone. It may be possible that intermolecular factors, such as chain packing, contribute to the higher indices observed in this particular polymer system. Further investigations are being undertaken to arrive at an explanation for this inconsistency. 


\subsection{UV-visible transmission}

Frequently, the increase in refractive index also leads to an increase in absorbance. This is manifested in the transmission data presented in Figure 2. The fluorenyl groups that are desirable for achieving higher refractive indices also lead to lower transmission values, especially in the case of BPFPA/FDA. However, other factors also seem to be at work in influencing transmission, as can be seen from BPADA/mBAPS and OptiNDEX ${ }^{\mathrm{TM}}$ B 44 . The near-UV transmission is much improved as a result of simply using the $m$-isomer versus the $p$-isomer in this case. Presumably, the resulting larger kinks in the $m$-isomer provide for greater void volumes in the packed film, while the overall polarizability is unaffected. While it is expected that larger void volumes exist in the BPFPA/FDA polyetherimide system as a result of the bulky spirocyclic fluorenyl units in the dianhydride and diamine, this does not lead to an improvement in the observed transparency in its film. In fact, the opposite is observed, where there is a pronounced decrease in transmission across the spectrum of interest. In this particular system, three-dimensional packing effects such as intrachain and interchain charge transfer complex (leading to the formation of highly absorbing transient species) formation may be particularly important.

\section{CONCLUSIONS}

The development of robust polymer coating systems that provide a defined range of refractive indices from 1.60 and greater has not been feasible because of the inherently low refractive index properties of many organic polymers. Our investigations into polyetherimide systems has shown that a reasonable variety of solvent-soluble systems can be synthesized and applied to make optical-quality films. In general, these films possess very good transparency in the visible region. Moreover, the imidized nature of the polymer solutions afford a means of minimizing film defects arising from polyimide film curing. It is simply necessary to ensure complete solvent removal in order to prepare the films. An additional advantage of having the polyetherimide in solution is that the shelf life is extended and cold storage, common with poly(amic acid) precursors, becomes completely unnecessary.

The highly aromatic nature of these polyetherimides provides most of the desirable characteristics found in polyimides, such as high strength and flexibility, excellent thermal stability, and fairly high glass transition temperatures. Through judicious utilization of a variety of functional groups that can be incorporated into the polyetherimide backbone, optical films of a predetermined range of refractive indices can be obtained.

\section{REFERENCES}

1. Eichstadt, A.E., T.C. Ward, M.D. Bagwell, I.V. Farr, D.L. Dunson, and J.E. McGrath, "Synthesis and Characterization of Amorphous Partially Aliphatic Polyimide Copolymers Based on Bisphenol-A Dianhydride," Macromolecules, 35, 2002, pp. 7561-7568.

2. Flaim, T.D., Y. Wang, and R. Mercado, "High Refractive Index Polymer Coatings for Optoelectronics Applications," Proceedings of SPIE: Optical Systems Design 2003, vol. 5250, Saint-Etienne, France, Sept. 29-Oct. $3,2003$.

3. Hasegawa, M., and K. Horie, "Photophysics, photochemistry, and optical properties of polyimides," Progress in Polymer Science, 26, 2001, pp. 259-335.

4. Farr, I.V., Synthesis and Characterization of Novel Polyimide Gas Separation Membrane Material Systems, Ph.D. Dissertation, 1999, Virginia Polytechnic Institute and State University, Blacksburg, VA. 